\title{
Chemical composition, antioxidant activity and bioactive compounds of vegetation species ingested by goats on semiarid rangelands
}

\author{
H.M. Cuchillo ${ }^{1,2}$, D.C. Puga ${ }^{1,6}$, N. Wrage-Mönning ${ }^{3}$, M.J.G. Espinosa ${ }^{4}$, B.S. Montaño', \\ A. Navarro-Ocaña ${ }^{4}$, J.A. Ledesma ${ }^{5}$, M.M. Díaz ${ }^{1}$ and R.F. Pérez-Gil ${ }^{1}$ \\ ${ }^{1}$ Animal Nutrition Department, National Institute of Medical Sciences and Nutrition "Salvador Zubirán"; D.F, 14000, Mexico \\ ${ }^{2}$ Tropical Forages Program, International Center for Tropical Agriculture (CIAT); Cali, 6713, Colombia \\ ${ }^{3}$ Faculty of Life Sciences, Agricultural Sciences, Rhine-Waal University of Applied Sciences; Kleve 47533, Germany \\ ${ }^{4}$ Faculty of Chemistry, National Autonomous University of Mexico (UNAM); D.F, 04510, Mexico \\ ${ }^{5}$ Direction of Nutrition, National Institute of Medical Sciences and Nutrition "Salvador Zubirán"; DF, 14000, Mexico
}

KEY WORDS: plant bioactives, free radical scavenging, antioxidant activity, semiarid vegetation, goats
Received: 9 July 2012

Revised: 17 May 2013

Accepted: 10 June 2013

${ }^{6}$ Corresponding author: e-mail: claudia.delgadillop@quetzal.innsz.mx

\begin{abstract}
The first goal of this study was to evaluate the chemical composition of vegetation consumed by goats on semiarid rangelands. Secondly, antioxidant activity and identified bioactive compounds of this vegetation were estimated. Twenty-five samples were analysed, including leaves, stems, fruits, or a combination of them. Chemical composition demonstrated large differences among species and smaller differences within species. Some species had high protein contents, e.g. Acacia schaffneri, Celtis pallida and Prosopis laevigata. Methanol:water was the best solution to extract bioactive compounds, which we found in larger amounts in complete plants and stems than in fruits and leaves. A. farnesiana pods had the largest polyphenol contents, whereas A. farnesiana, A. schaffneri, Leptochloa dubia and others showed the best antioxidant activity. A positive correlation was observed between antioxidant activity and polyphenol-flavonoid concentration. Based on these results, it seems necessary to study in more detail some species, e.g. the fruits of Acacia farnesiana and $A$. schaffneri, to better understand their implications for ruminant feeding and nutrition.
\end{abstract}

\section{Introduction}

In 2009, approximately 270 million goats in the least developed countries around the world were maintained mostly in arid and semi-arid ecosystems based on available local fodder resources (FAOSTAT, 2010). In these developing countries, where the demand for food supplies is growing due to population growth, this kind of farming increases food security. Goats are especially suited for such management as they are able to maintain a reasonable output even when the forage on offer is scarce or when it comprises poor quality feedstuffs (Jiménez-Ferrer et al., 2008). Plant species in these conditions resist herbivore ingestion by biological strategies, principally through production of plant bioactive compounds, which might considerably change animal-plant interrelationships (Villalba et al., 2010; Wrage et al., 2011) and potentially reduce the digestibility of the diet (Rochfort et al., 2008).

Some authors have suggested that pastoral feeding on shrublands may increase the animals' wellbe- 
ing (Rochfort et al., 2008; Vasta et al., 2008; Patra and Saxena, 2011). For example, Acacia pennatula has been shown to have anthelmintic properties due to its plant bioactive compounds (PBC) (AlonsoDíaz et al., 2010). Complementary effects of PBC, e.g. flavonoids, have been claimed to include antiinflammatory, antiallergic, antimicrobial, anticarcinogenic activities, cholesterol modulation, and reduction of the incidence of cerebral infarctions. Hydroxycinnamic acids have shown analogous beneficial activities (Vemuri et al., 2008). It is necessary, however, to consider both the benefits and disadvantages of ingestion of PBC. Some inconveniences of PBC ingestion are the detrimental effects on the digestibility of feedstuffs and worse animal performance (Ben Salem et al., 2005).

Nutraceutical properties transferred from plant tissues to ruminants are expected to influence animal products for human consumption (Rochfort et al., 2008); for instance, grazing or browsing have demonstrated to enhance the quality of meat, milk and cheese products more than their basic nutritional parameters (Vasta et al., 2008; Pajor et al., 2009; Cuchillo et al., 2010a,b).

Studies investigating PBC have focussed on temperate forages, legumes and seeds or on tropical plant species (Carnachan and Harris, 2000; Mustafa et al., 2010). The knowledge of feedstuffs from arid and semiarid ecosystems is much more limited with respect to their PBC contents and antioxidant activities. Usually, investigations of arid or semiarid systems have mainly evaluated nutritional characteristics of a few vegetation species, mainly herbs. Moreover, the assessments that included shrub species have mostly tested these for detrimental feed ingredients and their impact on dry matter intake and protein digestibility (Ben Salem et al., 2005; Baraza et al., 2008; GarcíaWinder et al., 2009).

Several studies have highlighted that the medium used to extract PBC can modify the biological activity of the analysed samples (Ruiz-Terán et al., 2008; Mustafa et al., 2010). Although methanol is the solvent most used for plant tissue extractions, other solvent systems have been used sucessfully to evaluate antioxidant activities and phenolic compounds (Alonso-Díaz et al., 2010; Reynaud et al., 2010). Solvents have not been compared for their potential to recover PBC for antioxidant activity assessment in rangeland plants. The goals of the present experiment were to evaluate the chemical composition, antioxidant activity, and bioactive compounds of plants consumed by goats on semiarid rangelands of central Mexico.

\section{Material and methods}

\section{Animals and experimental design}

The experiment was carried out in Queretaro, Mexico $\left(20^{\circ} 35^{\prime} \mathrm{N}, 100^{\circ} 18^{\prime} \mathrm{W}\right.$; 1,950 m.a.s.1.) during the summer of 2008. The area has a dry, semiarid climate with an average annual precipitation of $460 \mathrm{~mm}$ with isolated rains in winter. A group of 40 French Alpine goats that weighed $50 \pm 5 \mathrm{~kg}$ and had a lactation period of 150 days was allowed to graze and browse freely from 08.00 to $17.00 \mathrm{~h}$ on 14 ha of rangeland. The animals were kept in overnight confinement and they did not receive any supplementary feeding. The vegetation included the following forbs, leguminous trees and cactaceous species: Acacia farnesiana, A. schaffneri, Aristida adsencionis, Bouteloua curtipendula, B. repens, Celtis pallida, Chloris virgata, Jatropha dioica, Leptochloa dubia, Mimosa biuncifera, Opuntia affasiacantha, O. amyctaea, O. hytiacantha, O. robusta, O. streptacantha, O. tomentosa, Prosopis laevigata, Rhynchelytrum roseum, Urochloa fasciculata, Verbasina serrata and Zalazania augusta. Sampling of grazed or browsed species was performed simulating the goats' bites, following the recommendations of Agreil and Meuret (2004). Briefly, two control animals were selected to be monitored continuously along intervals of $30 \mathrm{~min}$ from a very close distance. The selected animals were adapted to the presence of observers to permit them to see the animal's mouth and the chosen plants without modifying the grazing behaviour. Observations of goats were carried out between August and September. Twentyfive rangeland vegetation samples were identified, collected and dried on 3 different days. Sampling was done twice daily. A total of one hundred and fifty samples was collected. The vegetation sampling included leaves, stems (cladodes for Opuntia species), fruits or a combination of them, in line with the individual bites of the goats.

\section{Plants' chemical composition}

The samples were ground to a particle size of $1 \mathrm{~mm}$ and analysed as follows: moisture (oven-drying at $60^{\circ} \mathrm{C}$ ), fat, crude fibre and ash contents were determined using standard methods (AOAC, 2003). Nitrogen was measured using the Micro-Kjeldahl technique (AOAC, 2003). N-free extractives were calculated as the difference between $100 \%$ and protein (nitrogen factor: 6.25), fat, crude fibre, and ash percentages. Gross energy was determined using the calorimetric Parr bomb (Parr Instrument Company, Illinois. USA). All samples were analysed in triplicate. 


\section{Extraction}

Three subsamples (20 g each) weighed in Erlenmeyer flasks with $150 \mathrm{ml}$ of methanol, methanol:water (80:20), or acetone, were set to shaking for $24 \mathrm{~h}$ at room temperature. All extracts were then filtered and washed (50 $\mathrm{ml}$ of respective solvent), then the filtrates were concentrated with a vacuum rotary evaporator (Büchi R-205, Labortechnik AG, Switzerland) at $30^{\circ} \mathrm{C}$ and $150 \mathrm{rpm}$. Further, extracts were frozen at $-80^{\circ} \mathrm{C}$, lyophilized (Labconco Freezone 6, Labconco Corp., Kansas City, MO, USA) and stored at $4{ }^{\circ} \mathrm{C}$ for later analysis.

\section{Qualitative radical scavenging activity}

Fifty milligrams of dry extracts were diluted with $0.5 \mathrm{ml}$ of the respective extraction medium. Afterwards, $20 \mu \mathrm{l}$ aliquots were applied individually to the baseline of TLC (thin layer chromatography) plates $(20 \times 10 \mathrm{~cm}$ silica gel 60 F254; Merck, Germany) and the sample was allowed to dry. Then, the TLC plates were eluted with a solvent system consisting of methanol:ethyl acetate (70:30). Once dried at room temperature, the plates were tested against $\mathrm{DPPH}^{+}$(1,1-diphenyl-2-picrylhydrazyl; 200 $\mathrm{mg} \mathrm{DPPH}^{+}$dissolved in $100 \mathrm{ml}$ methanol) spray reagent to determine the qualitative radical scavenging activity (QLRA) of the samples, visible as yellow-on-purple spots due to the decoloration of $\mathrm{DPPH}^{+}$(Sharma et al., 1998). Butylhydroxyanisol (BHA) and $\alpha$-tocopherol standards were used as references.

\section{Quantitative radical scavenging activity (QRA)}

QRA was determined following the method of von Gadow et al. (1997) with some modifications. Briefly, $0.25 \mathrm{ml}$ of each extract solution (200 ppm) were added to $2 \mathrm{ml} 0.36 \mathrm{mM} \mathrm{DPPH}^{+}$solution. The mixture was shaken vigorously and left to stand for $30 \mathrm{~min}$ in the dark. Absorbance was measured at $517 \mathrm{~nm}$ at $t=0$ and after 30 min using a Beckman DU-70 spectrophotometer. Quantitative radical scavenging activity was calculated as follows: QRA $(\%)=\left(\left(A t_{0}-A t_{\text {end }}\right) / A t_{0}\right) * 100$, where: $A t_{0}$ is the initial absorbance at time zero and $A t_{\text {end }}$ is the final absorbance after $30 \mathrm{~min}$. BHA and $\alpha$-tocopherol standards (100 ppm) were used as references. All determinations were performed three times on each sample.

\section{Total polyphenol content and plant bioactive compounds (PBC)}

Total polyphenol content (TPC) in the plant extract (methanol:water $(80: 20 \mathrm{v} / \mathrm{v})$ ) was determined by the Folin-Ciocalteu colorimetric method described by Taga et al. (1984). The concentration was calculated using gallic acid as the standard, and the results were expressed as $\mathrm{mg}$ of gallic acid equivalents (GAE) per $1 \mathrm{~kg}$ dry matter of plant extract. To determine flavonoids and hydroxycinnamic acid, $20 \mathrm{mg}$ of each dry extract were assessed by HPLC according to Ubando-Rivera et al. (2005). An HPLC 1525 high-pressure binary pump (Waters Milford, USA) and Symmetry C18 column (5 $\mu$ m steel 3.9 $\mathrm{mm} \times 150 \mathrm{~mm}$; Waters Milford, USA) were employed. Methanol:water (at a ratio of 70:30 v/v) and $0.16 \mathrm{M}$ acetic acid (pH 2.4) were used as carriers at a flow rate of $1 \mathrm{ml} \cdot \mathrm{min}^{-1}$. The oven temperature was held at $45^{\circ} \mathrm{C}$, whereas the detection was performed at $280 \mathrm{~nm}$ (486 Waters Milford, USA). The following substances dissolved in methanol were used as standards: catechin $\left(2.21 \mathrm{mg} \cdot \mathrm{ml}^{-1}\right)$, epicatechin $\left(1.25 \mathrm{mg} \cdot \mathrm{ml}^{-1}\right)$, gallocatechin $\left(0.032 \mathrm{mg} \cdot \mathrm{ml}^{-1}\right)$, gallic acid $\left(0.030 \mathrm{mg} \cdot \mathrm{ml}^{-1}\right)$, caffeic acid $(0.032$ $\left.\mathrm{mg} \cdot \mathrm{ml}^{-1}\right)$, cinnamic acid $\left(0.038 \mathrm{mg} \cdot \mathrm{ml}^{-1}\right)$ and ferulic acid $\left(0.031 \mathrm{mg} \cdot \mathrm{ml}^{-1}\right)$. Calibration curves were made for each standard using three dilutions $(1: 1 ; 1: 3$ and $1: 5)$. Peaks were identified by the retention times of individual standard flavonoids and hydroxycinnamic acids, using Brezze version 6.30 Waters Software. The concentrations of hydroxycinnamic acids were expressed as $\mathrm{g} \cdot 100 \mathrm{~g}^{-1}$, while flavonoid concentrations were expressed as $\mathrm{mg} \cdot 100 \mathrm{~g}^{-1}$ dry matter of extract. All analytical reagents and standards were from SigmaAldrich, Steinheim, Germany.

\section{Statistical analysis}

Chemical composition, total polyphenol content, hydroxycinnamic acids, flavonoids, and quantitative radical scavenging activity (QRA) of plant samples were analysed by ANOVA $(p=0.05)$ using SAS (2003). The days of collection were treated as repeated measurements. The results of the parallel measurements of each individual sample were averaged before further statistical analysis. For qualitative radical scavenging activity we used nonparametric statistics. The Friedman test was used to establish differences among extract responses; further, the Wilcoxon signed ranks test for related samples was used to identify such differences. For each plant portion (complete, fruits, leaves and stems; cladodes for Opuntia species) we used the nonparametric statistic of $\mathrm{K}$ independent samples. The Kruskal-Wallis test was used to stablish differences among plant portions. Further, the Mann-Whitney U signed ranks test for related pairs of portions was used to identify such differences (SPSS, 2010). 
Table 1. Chemical composition of shrub species browsed or grazed by goats on semiarid rangelands, $\mathrm{g} \cdot 100 \mathrm{~g}^{-1}$ dry-weight basis

\begin{tabular}{|c|c|c|c|c|c|c|c|c|c|}
\hline pecies & rtion & $P$ & $\mathrm{H}$ & $E$ & F & E & DN & al & ial \\
\hline $\begin{array}{l}\text { Aristida } \\
\text { adsencionis }\end{array}$ & & $71+035$ & \pm 0.28 & $8 \pm 0.08$ & $2^{\mathrm{k}} \pm 0.27$ & $7^{\mathrm{h}} \pm 0.67$ & \pm 0.78 & 03 & $2.3^{\mathrm{dc}} \pm 0.03$ \\
\hline $\begin{array}{l}\text { Acacia } \\
\text { schaffneri }\end{array}$ & $\ddagger$ & 1 & 1 & 6 & 2 & 13 & 8 & $3.0^{b} \pm 0.01$ & 0 \\
\hline $\begin{array}{l}\text { Bouteloua } \\
\text { curtipendula }\end{array}$ & molete & $6.5^{n} \pm 0.17$ & $9.2 \pm 0.11$ & $4 \pm 0.09$ & $.1^{1 \pm} \pm 0.11$ & $.7^{\mathrm{m}} \pm 0.08$ & 62.1 & $7^{\mathrm{d}} \pm 0.01$ & $.2^{\mathrm{dc}} \pm 0.01$ \\
\hline aut & & $3^{m} \pm 0.06$ & & & $7.3 \pm 0.27$ & $2.7^{\circ} \pm 0.11$ & $61.1^{n} \pm 0.33$ & $27 \mathrm{~d}+001$ & 2de+s d \\
\hline Chlori & 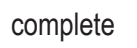 & 13 & 16 & & & & & . & \\
\hline 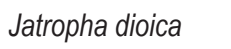 & & & & & & & & & \\
\hline Leptochloa o & & & & & & & & & \\
\hline Mimosa biur & te & $193 b$ & 14 & 11 & .16 & 13 & & & 0.05 \\
\hline & & & & & & & & & \\
\hline fasciculata & & 6 & 10 & 9 & 45 & 15 & 5 & 2 & .51 \\
\hline Mean complete & & $9.27^{A} \pm 0.16$ & $8.89^{A_{-}}$ & $1.77^{\mathrm{A}}$ & $24.56^{\mathrm{A}} \pm 0.45$ & $55.7^{\mathrm{A}_{ \pm}}$ & 63 & & .51 \\
\hline Avia lai & & & & & & & & & \\
\hline Opur & fruits & & & & & & & & \\
\hline hytiacal & (1) & & & & & & & & 5 \\
\hline rosopis lae & & 1 & & & & 6 & 6 & & \\
\hline M & & & & & & & & & \\
\hline cellis & & & 1 & & & & & & \\
\hline Prosopis lae & 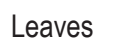 & 1 & 23 & 2.1 & 32 & 50 & & 5 & \\
\hline V & (2) & & 12 & $1.1-0.10$ & & & & & \\
\hline Mear & & 1 & 1 & 1.64 & & & & & 0.05 \\
\hline Celtis & (110 & & & & & & & & .05 \\
\hline Verba & stems & & & & & & & & \\
\hline a & & & & & & & & & \\
\hline S teins & & & $5.4^{\mathrm{D}} \pm 2.1$ & & & & & & \\
\hline $\begin{array}{l}\text { Opuntia } \\
\text { affasiacanth }\end{array}$ & odes & $4.0^{t} \pm 0.11$ & 2 & 1.1 & & 18 & 56. & 2.4 & 05 \\
\hline $\begin{array}{c}\text { Opuntia } \\
\text { hytiacar }\end{array}$ & les & & & \pm 0.18 & 17 & & 53 & & 05 \\
\hline Opuntia robusta & cladodes & & $r$ & $1.6^{5}$ & & 64 & & & \\
\hline $\begin{array}{l}\text { Opuntia } \\
\text { streptacantha }\end{array}$ & oues & 4 & 37 & .17 & \pm 0.16 & $53.5^{n} \pm 0.11$ & 22 & 5 & 0.05 \\
\hline $\begin{array}{l}\text { Opuntia } \\
\text { tomentosa }\end{array}$ & adodes & & 28 & & $10.4 \pm$ & & 98 & & 0.05 \\
\hline Mean cladodes & & $5.9^{\mathrm{E}} \pm 1.75$ & $23.6^{\mathrm{E}} \pm 1.21$ & $1.7^{\mathrm{E}} \pm 0.17$ & $14.0^{\mathrm{E}} \pm 4.31$ & $54.6^{\mathrm{E}} \pm 3.6$ & $55.2^{\mathrm{E}} \pm 1.25$ & $2.4^{E} \pm 0.18$ & $2.0^{E} \pm 0.18$ \\
\hline
\end{tabular}

$\mathrm{DM}$ - dry matter; $\mathrm{CP}$ - crude protein $(\mathrm{N} \times 6.25)$; $\mathrm{EE}$ - ether extract; $\mathrm{CF}$ - crude fibre; NFE - N-free extractives; TDN - total digestible nutrients $(\mathrm{TDN}=\mathrm{CF}(0.5)+\mathrm{CP}(0.75)+\mathrm{NFE}(0.75)+(\mathrm{EE}(0.75) \times 2.25)) ; \mathrm{DE}$ - digestible energy (TDN x 4409 kcal); ME - metabolizable energy (DE $\times 0.82)$.

Means with different superscript letters within columns are significantly different at $p=0.05$. Complete $=$ mix of leaves and stems; $\ddagger-\operatorname{mix}$ of leaves, stems and pods; Means with capital letters are significantly different $\left({ }^{*} p=0.05,{ }^{* *} P=0.001\right.$ ) within column where:

$C P: A \neq C^{* *}, A \neq E^{* *}, B \neq C^{* *}, C \neq D^{* *}, C \neq E^{* *}$

ASH: $A \neq B^{* *}, A \neq C^{* *}, A \neq D^{* *}, A \neq E^{* *}, B \neq C^{* *}, B \neq E^{* *}, C \neq D^{* *}, C \neq E^{* *}, D \neq E^{* *}$

$E E: A \neq B^{* *}, B \neq E^{* *}$

$C F: A \neq C^{* *}, A \neq D^{* *}, A \neq E^{* *}, C \neq D^{* *}, D \neq E^{* *}$

NFE: $A \neq B^{* *}, A \neq D^{* *}, B \neq C^{* *}, B \neq D^{* *}, B \neq E^{* *}, C \neq D^{*}, D \neq E^{* *}$

TDN: $A \neq B^{* *}, A \neq C^{*}, A \neq E^{* *}, B \neq D^{*}, B \neq E^{*}, C \neq E^{* *}, D \neq E^{* *}$

$D E: A \neq B^{*}, A \neq E^{* *}, B \neq D^{*}, B \neq E^{* *}, C \neq E^{* *}, D \neq E^{* *}$

ME: $A \neq B^{*}, A \neq E^{* *}, B \neq D^{*}, B \neq E^{* *}, C \neq E^{* *}, D \neq E^{* *}$ 
Table 2. Qualitative (QLRA) and quantitative (QRA) radical scavenging activity by $\mathrm{DPPH}^{+}$radical ${ }^{\S}$ essay of endemic vegetation species browsed or grazed by goats on semiarid rangelands

\begin{tabular}{|c|c|c|c|c|c|c|}
\hline \multirow[t]{2}{*}{ Species } & \multicolumn{3}{|c|}{ QLRA by TLC ${ }^{\dagger}$} & \multicolumn{3}{|l|}{ QRA \% } \\
\hline & $\mathrm{MeOH}$ & $\mathrm{MeOH}: \mathrm{H}_{2} \mathrm{O}(80: 20)$ & acetone & $\mathrm{MeOH}$ & $\mathrm{MeOH}: \mathrm{H}_{2} \mathrm{O}(80: 20)$ & acetone \\
\hline Aristida adsencionis & +++ & +++ & + & $26.43^{\mathrm{ac}} \pm 0.84$ & $24.34^{\mathrm{bG}} \pm 0.46$ & $11.22^{\mathrm{CDE}} \pm 0.19$ \\
\hline Acacia schaffneri & ++++ & ${ }^{++++}$ & ++ & $42.08^{\mathrm{bB}} \pm 0.14$ & $47.38^{\mathrm{aA}} \pm 0.17$ & $6.46^{\mathrm{CFGH}} \pm 2.20$ \\
\hline Bouteloua curtipendula & +++ & +++ & + & $25.09^{b c} \pm 0.63$ & $35.42^{\mathrm{aD}} \pm 0.24$ & $18.52^{\mathrm{CAB}} \pm 2.23$ \\
\hline Bouteloua repens & ++ & +++ & + & $17.80^{\mathrm{bDE}} \pm 0.02$ & $30.92^{\mathrm{aBC}} \pm 0.02$ & $8.55^{\mathrm{CFGH}} \pm 0.01$ \\
\hline Chloris virgata & +++ & ++ & + & $27.12^{\mathrm{ac}} \pm 0.38$ & $27.39^{\mathrm{al}} \pm 0.61$ & $16.6^{\mathrm{bBC}} \pm 1.56$ \\
\hline Jatropha dioica & ++ & ++ & +++ & $10.03^{\mathrm{bJH}} \pm 0.09$ & $24.13^{\mathrm{a} G} \pm 0.61$ & $5.79^{\mathrm{CF} F \mathrm{GH}} \pm 1.13$ \\
\hline Leptochloa dubia & +++ & ++ & + & $47.01^{\mathrm{aA}} \pm 1.84$ & $21.48^{\mathrm{bH}} \pm 0.67$ & $14.58^{\mathrm{CDC}} \pm 0.87$ \\
\hline Mimosa biuncifera & ++ & ++ & +++ & $8.93^{\mathrm{blJ}} \pm 0.27$ & $28.70^{\mathrm{aFE}} \pm 2.04$ & $5.67^{\mathrm{CFIGH}} \pm 0.67$ \\
\hline Rhynchelytrum roseum & ++++ & +++ & + & $40.08^{\mathrm{bB}} \pm 0.19$ & $44.63^{\mathrm{aBC}} \pm 0.24$ & $6.44^{\mathrm{CFGH}} \pm 0.35$ \\
\hline Urochloa fasciculata & +++ & ++ & + & $20.11^{\mathrm{bD}} \pm 0.93$ & $30.19^{\mathrm{aE}} \pm 0.46$ & $5.95^{\mathrm{CFIGH}} \pm 0.49$ \\
\hline Mean complete & & & & $26.47^{A^{*}} \pm 12.6$ & $31.46^{\mathrm{A}^{*}} \pm 8.36$ & $9.98^{A^{*}} \pm 4.87$ \\
\hline Acacia farnesiana & t+++ & ${ }_{++++}$ & +++ & $43.22^{\mathrm{bAB}} \pm 0.15$ & $47.59^{\mathrm{aA}} \pm 0.13$ & $20.78^{\mathrm{CA}} \pm 1.64$ \\
\hline Opuntia amyctaea & ++ & + & + & $12.11^{\mathrm{bHG}} \pm 0.36$ & $20.83^{\mathrm{aH}} \pm 0.36$ & $4.00^{\mathrm{ClGH}} \pm 1.59$ \\
\hline Opuntia hytiacantha & ++ & ++ & + & $10.84^{\mathrm{bHG}} \pm 0.02$ & $18.30^{\mathrm{al}} \pm 0.33$ & $5.57^{\mathrm{CFIGH}} \pm 0.72$ \\
\hline Prosopis laevigata & + & ++ & + & $14.72^{\mathrm{bEGF}} \pm 4.5$ & $22.92^{\mathrm{aHG}} \pm 0.95$ & $2.70^{\mathrm{clH}} \pm 1.52$ \\
\hline Mean fruits & & & & $20.22^{A B^{+}} \pm 14.0$ & $27.41^{B^{*}} \pm 12.3$ & $8.26^{\mathrm{B}^{+}} \pm 7.72$ \\
\hline Celtis pallida & ++ & ++ & ++ & $10.41^{\mathrm{bJHG}} \pm 0.52$ & $29.49^{\mathrm{aEF}} \pm 0.29$ & $4.87^{\mathrm{CFIGH}} \pm 0.51$ \\
\hline Prosopis laevigata & +++ & +++ & ++ & $19.99^{\mathrm{bD}} \pm 0.28$ & $35.68^{\mathrm{aD}} \pm 1.24$ & $7.36^{\mathrm{CFG}} \pm 0.64$ \\
\hline Verbasina serrata & + & ++ & + & $6.10^{\mathrm{bJ}} \pm 0.09$ & $17.04^{\mathrm{al}} \pm 0.22$ & $2.50^{\mathrm{cl}} \pm 0.52$ \\
\hline Mean leaves & & & & $12.17 c^{*} \pm 6.17$ & $27.40^{B^{*}} \pm 8.25$ & $4.91^{D^{*}} \pm 2.16$ \\
\hline Celtis pallida & + & + & + & $17.06^{\mathrm{bDE}} \pm 0.90$ & $44.02^{\mathrm{ac}} \pm 1.54$ & $7.83^{\mathrm{CFE}} \pm 0.91$ \\
\hline Verbasina serrata & + & + & + & $12.69^{\mathrm{BEHGF}} \pm 1.5$ & $17.78^{\mathrm{al}} \pm 0.22$ & $5.66^{\mathrm{CFIGH}} \pm 1.14$ \\
\hline Zalazania augusta & ++ & +++ & + & $27.76^{\mathrm{bC}} \pm 3.56$ & $46.41^{\mathrm{aAB}} \pm 0.80$ & $6.97^{\mathrm{CFG}} \pm 0.84$ \\
\hline Mean stems & & & & $19.17^{A B^{*}} \pm 7.01$ & $36.07^{A^{*}} \pm 13.78$ & $6.82^{C^{*}} \pm 1.59$ \\
\hline Opuntia affasiacantha & + & + & ++ & $16.52^{\mathrm{bEDF}} \pm 0.50$ & $24.13^{\mathrm{aG}} \pm 0.86$ & $7.36^{\mathrm{CFG}} \pm 1.08$ \\
\hline Opuntia hytiacantha & + & + & ++ & $14.64^{\mathrm{bEHGF}} \pm 0.48$ & $46.92^{\mathrm{aA}} \pm 0.39$ & $6.93^{\mathrm{CFG}} \pm 0.80$ \\
\hline Opuntia robusta & + & + & + & $13.53^{\mathrm{BEHGF}} \pm 0.67$ & $16.14^{\mathrm{al}} \pm 0.91$ & $7.13^{\mathrm{cFG}} \pm 0.85$ \\
\hline Opuntia streptacantha & + & + & ++ & $16.57^{\mathrm{aEDF}} \pm 0.74$ & $16.39^{\mathrm{al}} \pm 0.04$ & $7.95^{\mathrm{bFE}} \pm 1.24$ \\
\hline Opuntia tomentosa & + & + & + & $14.26^{\mathrm{bEHGF}} \pm 2.08$ & $16.77^{\mathrm{al}} \pm 0.39$ & $6.21^{\mathrm{cFIGH}} \pm 1.47$ \\
\hline Mean cladodes & & & & $15.10^{c^{*}} \pm 1.51$ & $24.07^{\mathrm{BC}} \pm 11.82$ & $7.12^{C^{*}} \pm 1.08$ \\
\hline BHA th & +++++ & ${ }^{+++++}$ & ${ }^{+++++}$ & 90.22 & 86.36 & 88.08 \\
\hline Alpha tocopherol & +++++ & +++++ & +++++ & 91.07 & 84.97 & 89.42 \\
\hline
\end{tabular}

${ }^{\dagger}$ Radical scavenging activity of plant extracts on TLC plates, developed by methanol:ethyl acetate (70:30, v/v). ${ }^{+}-$weak intensity; ${ }^{++}-$intermediate intensity; ${ }^{+++}$- strong intensity, ${ }^{++++}$- very strong intensity. ${ }^{\S} \mathrm{DPPH}^{+}=1,1$-diphenyl-2-picrylhydrazyl $\left(200 \mathrm{mg} \mathrm{of} \mathrm{DPPH}{ }^{+} \cdot 100^{-1} \mathrm{ml}\right.$ methanol). 抄BHA - butylhydroxyanisol. Means with different small letters within the same row are significantly different at $p=0.05$. Means with different capital letters within columns are significantly different at $p=0.05$; " means values of plants portions within the different extractants are significantly different at $P=0.001,{ }^{A, B, C, D}$ means values of plants portions with different capital letters within columns are significantly different at $p=0.05$ 
Table 3. Total polyphenol, hydroxycinnamic acid and flavonoid content of endemic vegetation species browsed or grazed by goats

\begin{tabular}{|c|c|c|c|c|c|c|c|}
\hline \multirow{2}{*}{ Species } & \multirow{2}{*}{ TPC } & \multicolumn{3}{|c|}{ Hydroxycinnamic acids, $\mathrm{g} \cdot 100 \mathrm{~g}^{-1} \mathrm{DM}$} & \multicolumn{3}{|c|}{ Flavonoids, $\mathrm{mg} \cdot 100 \mathrm{~g}^{-1}$} \\
\hline & & gallic & caffeic & cinnamic & gallocatechin & catechin & epicatechin \\
\hline Aristida adsencionis & $160^{m} \pm 11.15$ & $0.162^{\mathrm{gfe}} \pm 0.001$ & $0.022^{f} \pm 0.004$ & $0.272^{f} \pm 0.004$ & $11.05^{\circ} \pm 0.865$ & $0.050^{e} \pm 0.015$ & $0.353^{d} \pm 0.045$ \\
\hline Acacia schaffneri & $2730^{b} \pm 30.2$ & $0.559^{c d} \pm 0.016$ & $0.200^{\mathrm{d}} \pm 0.017$ & ND & ND & $0.064^{e} \pm 0.017$ & $1.342^{b} \pm 0.111$ \\
\hline $\begin{array}{l}\text { Bouteloua } \\
\text { curtipendula }\end{array}$ & $314^{i g h} \pm 10.07$ & $0.194^{g f e} \pm 0.005$ & ND & $0.023^{h} \pm 0.007$ & $8.32^{f} \pm 0.840$ & $0.045^{e} \pm 0.021$ & $0.180^{\mathrm{e}} \pm 0.009$ \\
\hline Bouteloua repens & $3649 \pm 10.41$ & $0.580^{a} \pm 0.171$ & $0.071^{\mathrm{fe}} \pm 0.020$ & $1.382^{c} \pm 0.135$ & ND & $0.870^{\circ} \pm 0.116$ & $0.316^{d} \pm 0.008$ \\
\hline Chloris virgata & $474^{f} \pm 2.65$ & $0.175^{g f e} \pm 0.016$ & $0.022^{f} \pm 0.011$ & $0.034^{\mathrm{h}} \pm 0.014$ & $6.50^{i} \pm 0.555$ & $0.044^{e} \pm 0.005$ & $0.077^{\mathrm{feg}} \pm 0.012$ \\
\hline Jatropha dioica & $189^{\mathrm{k}} \pm 4.51$ & $0.106^{g f} \pm 0.16$ & $0.047^{f} \pm 0.018$ & ND & ND & $0.070^{e} \pm 0.022$ & $0.011^{g} \pm 0.001$ \\
\hline Leptochloa dubia & $146^{\mathrm{nlm}} \pm 8.5$ & $0.047^{\mathrm{cd}} \pm 0.013$ & $0.015^{f} \pm 0.003$ & $0.023^{h} \pm 0.012$ & $8.66^{e} \pm 1.149$ & $0.035^{e} \pm 0.011$ & $0.008^{9} \pm 0.001$ \\
\hline Mimosa biuncifera & $341^{\mathrm{gh}} \pm 2.52$ & $0.223^{\mathrm{fe}} \pm 0.0647$ & $0.020^{f} \pm 0.0037$ & $0.422^{\mathrm{e}} \pm 0.0088$ & $0.07^{n} \pm 0.0016$ & $1.105^{b} \pm 0.0866$ & $0.009^{9} \pm 0.0011$ \\
\hline $\begin{array}{l}\text { Rhynchelytrum } \\
\text { roseum }\end{array}$ & $231^{\mathrm{j} k} \pm 3.51$ & $0.255^{e} \pm 0.0397$ & $0.070^{f} \pm 0.0021$ & $0.020^{h} \pm 0.0044$ & $8.09^{9} \pm 0.1732$ & $0.004^{e} \pm 0.0009$ & $0.145^{e f} \pm 0.0208$ \\
\hline Urochloa asciculata & $966^{\circ} \pm 7.09$ & $0.048^{9} \pm 0.0173$ & $0.085^{\mathrm{fe}} \pm 0.0113$ & ND & ND & $0.021^{\mathrm{e}} \pm 0.0006$ & $0.050^{\text {feg }} \pm 0.0250$ \\
\hline Mean complete & $592^{\mathrm{A}} \pm 724$ & $0.253 \pm 0.036$ & $0.061 \pm 0.010$ & $0.311 \pm 0.026$ & $7.12 \pm 0.597$ & $0.231^{A} \pm 0.030$ & $0.249^{A} \pm 0.023$ \\
\hline Acacia farnesiana & $38170^{a} \pm 357$ & ND & ND & ND & ND & $0.004 \mathrm{e} \pm 0.0020$ & $0.003^{9} \pm 0.0013$ \\
\hline Opuntia amyctaea & $587^{e} \pm 23.4$ & ND & ND & ND & $1.150^{\mathrm{m}} \pm 0.1001$ & $0.004 \mathrm{e} \pm 0.0028$ & $0.021^{\mathrm{fg}} \pm 0.0015$ \\
\hline Opuntia hytiacantha & $343^{g h} \pm 4.58$ & $0.037^{g} \pm 0.0080$ & $0.031^{\dagger} \pm 0.0010$ & ND & $25.56^{\mathrm{a}} \pm 2.5494$ & $0.083^{e} \pm 0.0095$ & $0.091^{\mathrm{feg}} \pm 0.0040$ \\
\hline Proso & $4^{i g h} \pm 21.5$ & $0.410^{\mathrm{dc}} \pm 0.0660$ & $0.230^{d} \pm 0.0029$ & $0.223^{\mathrm{fg}} \pm 0.0300$ & $8.20^{f g} \pm 0.5519$ & $0.700^{d} \pm 0.0366$ & $0.530^{c} \pm 0.0502$ \\
\hline Mea & $9854^{\mathrm{B}} \pm 17077$ & $0.224 \pm 0.0370$ & $0.131 \pm 0.0019$ & $0.223 \pm 0.0300$ & $11.64 \pm 1.067$ & $0.198^{B} \pm 0.0509$ & $0.161^{\mathrm{B}} \pm 0.0571$ \\
\hline Celtis pallida & $280^{\mathrm{inh}} \pm 31.01$ & $0.090^{g f} \pm 0.0089$ & $0.021^{\dagger} \pm 0.0018$ & $0.040^{h} \pm 0.0052$ & ND & $0.004^{e} \pm 0.0006$ & $0.003^{9} \pm 0.0010$ \\
\hline Prosopis laevigata & 95 & ND & $1.065^{a} \pm 0.1407$ & $2.860^{b} \pm 0.2123$ & ND & $1.400^{\mathrm{a}} \pm 0.1229$ & $0.545^{c} \pm 0.0866$ \\
\hline Verbasina serrata & $272^{i j} \pm 15.53$ & $0.175^{\mathrm{efg}} \pm 0.0215$ & $0.380^{c} \pm 0.0917$ & ND & $10.21^{\mathrm{d}} \pm 0.5112$ & $0.004^{e} \pm 0.0006$ & $0.005^{f g} \pm 0.0002$ \\
\hline M & ${ }^{c} \pm 347$ & $0.133 \pm 0.0152$ & $0.489 \pm 0.0781$ & $1.45 \pm 0.1087$ & $10.21 \pm 0.5112$ & $0.469^{c} \pm 0.0413$ & $0.184^{C} \pm 0.0292$ \\
\hline Celt & 08 & $0.090^{g f} \pm 0.0089$ & ND & ND & $4.23 \pm 0.5323$ & $0.012^{\mathrm{e}} \pm 0.0025$ & $0.010^{9} \pm 0.0025$ \\
\hline Verbasina & $4^{\mathrm{d}} \pm 3$ & $0.061^{g} \pm 0.0171$ & $0.011^{f} \pm 0.0014$ & $0.030^{h} \pm 0$ & $20.13^{b} \pm 5.7160$ & $0.090^{\mathrm{e}} \pm 0.0091$ & $0.004^{9} \pm 0.0007$ \\
\hline \multirow[t]{2}{*}{ Zalazania augusta } & $480^{f} \pm 19.29$ & $0.510^{\mathrm{cb}} \pm 0.090$ & $0.044^{f} \pm 0.0040$ & $1.220^{d} \pm 0.1819$ & ND & $0.004^{e} \pm 0.0008$ & $0.003^{9} \pm 0.0009$ \\
\hline & $575^{\circ} \pm 231.06$ & $60.220 \pm 0.0386$ & $0.028 \pm 0.0027$ & $0.625 \pm 0.0952$ & $12.18 \pm 3.124$ & $0.035^{D} \pm 0.0041$ & $0.006^{D} \pm 0.0014$ \\
\hline Opuntia affasiacantha & a $202^{k} \pm 13.5$ & $0.044^{9} \pm 0.0045$ & $0.040^{f} \pm 0.0056$ & ND & $6.24 \pm 0.9103$ & $0.006^{e} \pm 0.0002$ & $0.008^{g} \pm 0.0010$ \\
\hline Opuntia hytiacantha & $77^{n} \pm 8.0$ & $0.090^{g f} \pm 0.0064$ & ND & ND & $4.42^{k} \pm 0.0252$ & $0.006^{e} \pm 0.0042$ & $0.003^{9} \pm 0.0007$ \\
\hline Opuntia robusta & $537^{\mathrm{ef}} \pm 21.46$ & $0.276^{\mathrm{de}} \pm 0.0203$ & $0.190^{\mathrm{de}} \pm 0.0046$ & $11.150^{a} \pm 0.0177$ & ND & $0.070^{\mathrm{e}} \pm 0.0002$ & $3.300^{\mathrm{a}} \pm 0.0809$ \\
\hline $\begin{array}{l}\text { Opuntia } \\
\text { streptacantha }\end{array}$ & $147^{\mathrm{nlm}} \pm 12.29$ & $90.202^{g f} \pm 0.0607$ & ND & ND & ND & $0.060^{\mathrm{e}} \pm 0.0192$ & $0.004^{g} \pm 0.0012$ \\
\hline Opuntia tomentosa & $335^{\mathrm{igh}} \pm 27.1$ & $0.060^{9} \pm 0.0105$ & $0.043^{f} \pm 0.0070$ & ND & $6.82^{h} \pm 0.8641$ & $0.020^{\mathrm{e}} \pm 0.0006$ & $0.013^{g} \pm 0.0010$ \\
\hline Mean cladodes & $259 \mathrm{E} \pm 169.0$ & $20.1347 \pm 0.0205$ & $0.091 \pm 0.0057$ & $11.15 \pm 0.0177$ & $5.83 \pm 0.5998$ & $0.0188^{A} \pm 0.0237$ & $0.665^{\mathrm{E}} \pm 0.0837$ \\
\hline
\end{tabular}

TPC - total polyphenol content ( $\mathrm{mg}$ of gallic acid equivalents (GAE) $\cdot \mathrm{kg}^{-1} \mathrm{dry}$ matter). ND - no detected; means with different letters within columns are significantly different at $p=0.05$; Means with capital letters are significantly different $\left({ }^{*} p=0.05,{ }^{*} P=0.001\right)$ within column where: TPC: $A \neq B^{*}, A \neq D^{*}, B \neq E^{* *}, D \neq E^{* *}$

Catechin: $A \neq E^{* *}$

Epicatechin: $A \neq D^{* *}, A \neq E^{*}, B \neq D^{*}$ 


\section{Results}

\section{Chemical composition}

The chemical composition of the analysed samples demonstrated large differences among plant species and smaller differences within species (Table 1). Shrubs showed large fibre and small crude protein $(\mathrm{CP})$ concentrations. Some species, however, had high crude protein values, e.g. Acacia schaffneri, Prosopis laevigata, Celtis pallida, Mimosa biuncifera and Verbasina serrata. Leaves normally have larger crude protein concentrations than fruits, stems, cladodes and complete plant samples. The largest values of metabolizable energy (ME) and total digestible nutrients (TDN) were found in A. farnesiana pods, whereas the largest crude fibre content was in Zalazania augusta stems. Variable results for ether extract, TDN and energy values were found among plant species and among plant parts. The significant differences between plant portions and pairs of portions found after the Kruskal-Wallis and Mann-Whitney U tests are shown in Table 1.

\section{Radical scavenging activity}

All of the extracts tested displayed qualitative radical scavenging activity (QLRA). Acetone extracts resulted in poorer responses than methanol $(P<0.003)$ and methanol:water $(P<0.001)$ extracts according to the Wilcoxon test. No difference was observed between methanol and methanol:water extracts when the Friedman test was employed $(p=0.157)$. Extracts of complete plants had a larger QLRA than fruits, leaves, stems, and cladodes, with some exceptions, e.g. Acacia fruits (Table 2). For QRA, whole plants yielded the best activity, followed by fruits, stems, and leaves. Differences were also observed, however, due to the extraction media employed; e.g., stems yielded higher radical protection measured in methanol:water than that observed in methanol and acetone extracts $(p<0.05)$. Cladodes tended to have the lowest mean for QRA for the three extractants. Our results revealed a close Pearson's correlation of the QRA measured by the $\mathrm{DPPH}^{+}$radical with total flavonoid $(\mathrm{r}=0.890)$ and TPC $(r=0.948)$ contents.

\section{Plant bioactive compounds}

Tests for PBC were performed only in methanolic extractions, since during the first QLRA screening, this alcoholic media showed a similar QLRA as methanol:water. Acetone extracts were discarded because they gave the lowest QLRA response among the three extractants. The analysis of different plant portions (complete plants, fruits, leaves, stems) resulted in different concentrations of PBC (Table 3).
Fruits had the highest mean value for total polyphenol content (TPC), whereas cladodes, the lowest, and this difference was significant $(P<0.001)$. This same relationship was observed when stems and cladodes were compared $(P<0.001)$. At single species level, $A$. farnesiana pods had the largest TPC $(38,170 \mathrm{mg}$ of $\mathrm{GAE} \cdot \mathrm{kg}^{-1} \mathrm{DM}$ ) of the shrubs (Table 3 ). The remaining extracts ranged from 77 (for O. hyatacantha cladodes) to $2730 \mathrm{mg}$ of GAE/kg MD (for $A$. schaffneri).

The results for hydroxycinnamic acids were variable among plant species and plant portions. For example, the analysis showed that the extract of Bouteloua repens (complete plant) had the largest gallic acid content $\left(0.580 \mathrm{~g} \cdot 100 \mathrm{~g}^{-1} \mathrm{DM}\right)$, whereas the leaves of $P$. laevigata contained the most caffeic acid $\left(1.065 \mathrm{~g} \cdot 100 \mathrm{~g}^{-1} \mathrm{DM}\right)$. O. robusta cladodes showed the highest cinnamic acid average $\left(11.15 \mathrm{~g} \cdot 100 \mathrm{~g}^{-1} \mathrm{DM}\right)$. For flavonoids, gallocatechin values of $O$. hyatacantha fruits $(25.56$

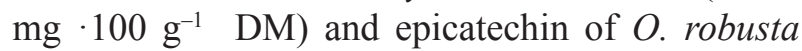
cladodes $\left(3.3 \mathrm{mg} \cdot 100 \mathrm{~g}^{-1} \mathrm{DM}\right)$ were the largest values measured. The complete plant mean epicatechin content was significantly different $(p<0.05)$ from the cladode mean; the same was found in epicatechin means between complete plants and stems. Complete plant and cladodes, and fruits, and stems were significantly different $(p<0.05)$. No significant difference was found in gallocatechin mean values after statistical analysis.

\section{Discussion}

In order to understand the potential benefits of rangeland plant communities for animal husbandry and production in Central Mexico, our first goal was to investigate the chemical composition of the vegetation. Results on composition indicated that the shrubland species are characterized by high fibre contents and poor protein values; these results are in line with other vegetation assessments from low precipitation areas (Baraza et al., 2008; García-Winder et al., 2009). Although Acacia, Mimosa, Prosopis and Verbasina species had high crude protein contents, the use of some of these resources might be limited due to the presence of large tannin contents, which are a part of PBC (Ben Salem et al., 2005; Jiménez-Ferrer et al., 2008). The threshold for tannins with no negative effects is about $50 \mathrm{~g} \cdot \mathrm{kg}^{-1}$, whereas larger ingestion would signify negative effects on intake. An intake of $20 \mathrm{~g} \cdot \mathrm{kg}^{-1}$, however, may have positive effects on protein metabolism, rumen eficiency, and animal performace (Patra and Saxena, 2011). 
Other researchers have pointed out that PBC contents are not the key factor for their consumption, instead, a high fibre content of feedstuffs seems to cause greater detrimental effects on intake (Alonso-Díaz et al., 2010). Nonetheless, at a moderate degree of consumption, such resources can be a valuable nutrient supply where seasonality and forage scarcity are the main limitations for low-input farming production. For example, the concentration of protein offered in the diet increased linearly with the inclusion of pods of the leguminous shrub $A$. farnesiana $(0 \%, 12 \%$, and $24 \%)$ in pelibuey lamb rations (García-Winder et al., 2009). VelázquezAvendaño et al. (2005) concluded that the inclusion of $40 \%$ A. farnesiana in wool-sheep diets based on maize straw is recommended as a valuable protein source.

Another important fodder resource, despite their low protein content (from 4.0 to $8.9 \%$ ), seemed to be Opuntia species, as their CP can be almost completely used to form microbial protein in the rumen. This is possible due to the readily available energy released from cladodes into ruminal fluid, which is essential for microbial protein synthesis (Parveen et al., 2010). The CP values of the Opuntia species in our study were around the $6 \%$ previously reported (Baraza et al., 2008; Parveen et al., 2010). Despite the good CP results of some of the shrub species, their exclusive use for goat feeding could cause temporary nutritional imbalances or permanent undernourishment, especially during dry seasons when grazing pressure is higher and the availability and nutritive value of forages decreases dramatically (García-Winder et al., 2009). To cope with this risk, proper supplementation during dry seasons is highly recommended (Galina et al., 2007).

In this context, food selection plays a key role in managing the nutritional demands for maintenance and productivity. The intake of specific plants and particular food items is regulated by the availability of the desired food and the capability of grazers to ingest and digest it (Reynaud et al., 2010; Villalba et al., 2010). Palatability, poisonous threshold, and the presence of alternative food choices are complementary causes that alter grazing behaviour; e.g., it is argued that plant bioactive compounds (PBC) modify animal behaviour and forage selection to maintain rumen functioning aimed at avoiding intoxication or metabolic disorders (Villalba et al., 2010). Therefore, the low content of PBC in leaves and cladodes may encourage their intake, whereas the high fibre and $\mathrm{PBC}$ contents of stems and complete plants may discourage their consumption (Patra and Saxena, 2011). Consequently, the total polyphenol content
(TPC) measured for A. farnesiana should be considered with special interest because of its large value $\left(38.2 \mathrm{~g}\right.$ of $\left.\mathrm{GAE} \cdot \mathrm{kg}^{-1}\right)$. Moreover, this concentration is similar to that reported by Ben Salem et al. (2005), who published values ranging from 37.3 to $69.8 \mathrm{~g} \mathrm{GAE} \cdot \mathrm{kg}^{-1}$ for $A$. cyanophylla leaves. In contrast, Alonso-Díaz et al. (2010) reported larger values for A. pennatula leaves $\left(97.2 \mathrm{~g} \mathrm{GAE} \cdot \mathrm{kg}^{-1}\right)$. Specific geographic conditions and the age of the sampled plants could play an important role in these discrepancies, as suggested by Baraza et al. (2008).

The ingestion of large quantities of such fodders may involve important changes in rumen physiology, for example, lower degradation rates of protein compromising rumen homeostasis or even animal health (Rochfort et al., 2008). Risky concentrations of TPC for animal consumption can be diminished by chopping or water spraying the feedstuffs before they are offered (Ben Salem et al., 2005). Generally, the efforts to avoid rumen health disorders or toxaemia are less important where diets include diverse botanical choices with variation of toxicity (Villalba et al., 2010). For example, Garcia-Winder et al. (2009) demonstrated that elevated contents of Acacia in lamb rations (24\%) can inhibit intake, while up to $12 \%$ of this shrub legume can be well tolerated and digested in combination with lucerne, maize, and soya-based feeding. In the present study, not all analysed plants showed high TPC values. In some species, these levels were similar to or lower than in species from temperate regions: e.g., $A$. adsencionis, $J$. dioica, $L$. dubia and $O$. streptocantha species had smaller TPC contents (from 0.147 to $0.189 \mathrm{~g}$ of $\mathrm{GAE} \cdot \mathrm{kg}^{-1}$ ) than three mixtures of French pasture plants that averaged $9.5 \mathrm{~g}$ of $\mathrm{GAE} \cdot \mathrm{kg}^{-1}$ (Reynaud et al., 2010). Dudonné et al. (2009) reported that the concentration of total phenolic compounds in 30 aqueous plant extracts ranged from 6.86 to $397.03 \mathrm{mg} \mathrm{GAE} \cdot \mathrm{g}^{-1}$ of sample measured by the Folin-Ciocalteu method, meanwhile we found values from 77 to $38170 \mathrm{mg} \mathrm{GAE} \cdot \mathrm{kg}^{-1}$ of sample DM. These differences could be a consequence of the extractant employed and nature of the plants, since these factors determine the potential antioxidant activity and PBC profile (Ruiz-Terán et al., 2008; Mustafa et al., 2010).

It is well known that the concentration of bioactive compounds alters the palatability of feedstuffs and, consequently, the feed intake. Nonethless, the significance of bioactive compounds has included benefical aspects such as the improvement in the yield and quality of ruminant-derived feeds (higher CLA content), control of parasites, lower ruminal amonnia $\mathrm{N}$ concentration, enhancement of microbial protein synthesis, lower incidence of pasture 
bloat, better live-weight gains, and mitigation of methane emisions (Rochfort et al., 2008; AlonsoDíaz et al., 2010; Patra and Saxena, 2011). In short, the inclusion of low amounts of $\mathrm{PBC}$ in animal diets has shown a positive impact on animal performance, milk and yield, without compromising dry matter intake or diet digestibilty. Furthermore, these PBCs are frequently cited as being the key to the underlying prevention and/or reduction of oxidative stress-related disorders (Quideau et al., 2011). Nevertheless, viewing plant polyphenols only as antioxidant agents must be considered with a great deal of caution, since the inclusion of high concentrations, which are still subjective values, affects palatability and feed intake and may cause poorer animal performance (Vasta et al., 2008). According to Quideau et al. (2011), the amount of PBC that can be found in each species depends on factors such as: plant resistance to microbial pathogens, resistance and tolerance to ingestion by herbivores, protection against solar radiation, reproduction phase of vegetation, plant nutrition, and interaction with other plants and organisms (insects, symbiotic fungi and bacteria).

The radical scavenging activity of $\mathrm{PBCs}$ is, in general, attributed to their hydroxyl groups, which determine their capabilities as effective antioxidants. According to the results of the present study, a positive trend between radical scavenging activity and bioactive compounds was confirmed (for TPC $r=0.948$, and for flavonoids, $r=0.890$ ). It has been suggested that a high correlation between these components is likely when complementary radical scavengers are absent and, at the same time, significant contents of TPC and flavonoids are present. In agreement with this finding, Mustafa et al. (2008) analysed plants by their antioxidant activity and their $\mathrm{PBC}$, demonstrating that such compounds contribute directly to this claimed benefit.

The capability of the extractants to recover PBC is also an important factor that largely determines the assessment of biological activities of extracts. In the current experiment, the qualitative radical scavenging activity (QLRA) and the quantitative radical scavenging activity (QRA) values for the same forage sample varied depending on the solvents used. Methanol:water generally showed higher QRA than methanol and acetone extracts, with a few exceptions; e.g., A. adsencionis and L. dubia. The results suggest that more polar components present in the methanol:water extracts contributed towards their increased measured scavenging activity. Water coupled with methanol could have an additive effect on hydrophilic antioxidants that methanol or acetone could not extract, thus being less capable to recover radical scavengers. In line with these results, RuizTerán et al. (2008) evaluated plant matrices by TLC and colorimetric assays, obtaining important variations in the responses when the solvent extract differed.

Our findings related to hydroxycinnamic acids showed that caffeic and cinnamic acids were not detected in any extract. A reason for this outcome could be that cell walls represent distinct and specific bound structural components of each plant (Komprda et al., 1999). Therefore, different cell wall components may change the digestibility of feedstuffs, since the hydroxycinnamic acids are in part responsible for the linkages between lignin and hemicellulose (Casler and Jung, 2006). The high value of cinnamic acid in $O$. robusta $(11.15 \%)$ may be related to this effect.

Recent findings suggest that a high concentration of plant metabolites in the rumen may modify the cellulolytic activity of bacteria by the formation of a complex among plant material, cell and bacteria membranes, and by diminishing protein degradation (Patra and Saxena, 2011). Rochfort et al. (2008) have indicated that a large presence of bioactive compounds is capable of causing direct depression of feed intake, which inhibits ruminal microorganisms and, therefore, causes lower weight gain in lambs. Parveen et al. (2010) proposed that rapid degradation of forages can lead to surplus excretion of nitrogen through urine due to a limited capacity of forming new microbial protein. In that case, the modulator effect of PBC could be beneficial in reducing nitrogen excretion while enchancing by-pass protein availability (Rochfort et al., 2008; Patra and Saxena, 2011). Despite most of the plants analyzed here having high fibre contents, the results permitted identification of valuable protein sources and contributed to filling in the gap in the knowledge about the bioactive compounds of scrubby vegetation and their significance in the diet of goats on semiarid areas. Furthermore, the selection activity of the goats and their ability to mix and match distinct feed items with different PBC contents indicate that they balance their PBC intake below the limit of harmful ingestion (Rochfort et al., 2008; Villalba et al., 2010). The chemical composition of the plant species and plant portions browsed and grazed by goats on semiarid rangelands, explains the presence of some PBCs in goat products (Vasta et al., 2008; Cuchillo et al., 2010a,b). 


\section{Conclusions}

Large protein contents of Acacia schaffneri, Celtis pallida, Mimosa biuncifera, and Prosopis laevigata lead these plants to being considered more valuable protein resources than before. Methanol:water was found to be the best solvent medium among those tested for extraction to assess radical scavenging activity. The antioxidant activity and plant bioactive compounds contents of rangeland vegetation are largely dependent on the plant species and plant part. Higher mean values of plant bioactive compounds were found in complete plants and stems than in fruits and leaves. A positive correlation was observed between radical scavenging activity and polyphenol-flavonoid concentration.

\section{References}

Agreil C., Meuret M., 2004. An improved method for quantifying intake rate and ingestive behaviour of ruminants in diverse and variable habitats using direct observation. Small Ruminants Res. $54,99-113$

Alonso-Díaz M.A., Torres-Acosta J.F., Sandoval-Castro C.A., Capetillo-Leal C.M., 2010. Polyphenolic compounds of nutraceutical trees and the variability of their biological activity measured by two methods. Trop. Subtrop. Agroecos. 12, 649-656

AOAC, 2003. Association of Official Analytical Chemists, Official Methods of Analysis. $17^{\text {th }}$ Edition, Washington, DC

Baraza E., Angeles S., Garcia A., Valiente-Banuet A., 2008. New natural resources as diet supplement for domestic goats during the dry season, in the Tehuacan Valley, Mexico. Interciencia 33, 891-896

Ben Salem H., Saghrouni L., Nefzaoui A., 2005. Attempts to deactivate tannins in fodder shrubs with physical and chemical treatments. Anim. Feed Sci. Tech. 122, 109-121

Carnachan S.M., Harris P.J., 2000. Ferulic acid is bound to the primary cell walls of all gymnosperm families. Biochem. Systemat. Ecol. 28, 865-879

Casler M.D., Jung H.-J.G., 2006. Relationships of fibre, lignin, and phenolics to in vitro fibre digestibility in three perennial grasses. Anim. Feed Sci. Tech. 125, 151-161

Cuchillo H.M., Puga D.C., Navarro O.A., Pérez-Gíl R.F., 2010a. Antioxidant activity, bioactive polyphenols in Mexican goats' milk cheeses on summer grazing. J. Dairy Res. 77, 20-26

Cuchillo H.M., Puga D.C., Wrage N., Pérez-Gíl R.F., 2010b. Feeding goats on scrubby Mexican rangeland and pasteurization Influences on milk and artisan cheese quality. Trop. Anim. Health. Prod. 42, 1127-1134

Dudonné S., Vitrac X., Coutière P., Woillez M., Mérillon J.-M., 2009. Comparative study of antioxidant properties and total phenolic content of 30 plant extracts of industrial interest using DPPH, ABTS, FRAP, SOD, and ORAC assays. J. Agr. Food Chem. 57, 1768-1774

FAOSTAT, 2010. Food and Agriculture Organization of the United Nations. 14th September 2010, http://faostat.fao.org/default.aspx

Galina M.A., Osnaya F., Cuchillo H.M., Haenlein G.F.W., 2007. Cheese quality from milk of grazing or indoor fed Zebu cows and Alpine crossbred goats. Small Ruminants Res. 71, 264-272

García-Winder L., Goñi-Cedeño S., Olguín-Lara P., Díaz-Salgado G., Arriaga-Jordán C., 2009. Huizache (Acacia farnesiana) whole pods (flesh and seeds) as an alternative feed for sheep in Mexico. Trop. Anim. Health. Prod. 41, 1615-1621
Jiménez-Ferrer G., López-Carmona M., Nahed-Toral J., OchoaGaona S., de Jong B., 2008. Fodder trees and shrubs of the north-tzotzil region of Chiapas. Mexico Vet. Méx. 39, 199-212

Komprda T., Stohandlová M., Foltyn J., Pozdisek J., Míka V., 1999. Content of $p$-coumaric and ferulic acid in forbs with potential grazing utilization. Arch. Tierernähr. 52, 95-105

Mustafa R.A., Hamid A.A., Mohamed S., Bakar F.A., 2010. Total phenolic compounds, flavonoids, and radical scavenging activity of 21 selected tropical plants, J. Food Sci. 75, C28-C35

Pajor F., Gallo O., Steiber O., Tasi J., Poti P., 2009. The effect of grazing on the composition of conjugated linoleic acid isomers and other fatty acids of milk and cheese in goats. J. Anim. Feed Sci. 18, 429-439

Parveen I., Threadgill M.D., Moorby J.M., Winters A., 2010. Oxidative phenols in forage crops containing polyphenol oxidase enzymes. J. Agr. Food Chem. 58, 1371-1382

PatraA.K., Saxena J., 2011. Exploitation of dietary tannins to improve rumen metabolism and ruminant nutrition. J. Sci. Food Agr. 91, 24-37

Quideau S., Deffieux D., Douat-Casassus C., Pouységu L., 2011. Plant polyphenols: chemical properties, biological activities and synthesis. Angew. Chem. Int. Ed. 50, 586-621

Reynaud A., Fraisse D., Cornu A., Farruggia A., Pujos-Guillot E., Besle J.-M., Martin B., Lamaison J.-L., Paquet D., Doreau M., Graulet B., 2010. Variation in content and composition of phenolic compounds in permanent pastures according to botanical variation. J. Agr. Food Chem. 58, 5485-5494

Rochfort S., Parker A.J., Dunshea F.R., 2008. Plant bioactives for ruminant health and productivity, Phytochemistry 69, 299-322

Ruiz-Terán F., Medrano-Martínez A., Navarro-Ocaña A., 2008. Antioxidant and free radical scavenging activities of plant extracts used in traditional medicine in Mexico. Afr. J. Biotechnol. 7, 1886-1893

SAS, 2003. Statistical Analysis System. User's Guide. SAS. Institute Inc. Cary, NC

Sharma O.P., Bhat T.K., Singh B., 1998. Thin-layer chromatography of gallic acid, methyl gallate, pyrogallol, phloroglucinol, catechol, resorcinol, hydroquinone, catechin, epicatechin, cinnamic acid, p-coumaric acid, ferulic acid and tannic acid. J. Chromatogr. 822, 167-171

SPSS, 2010. SPSS for Windows, v 18. SPSS Inc. Chicago III

Taga M., Miller E., Pratt D., 1984. Chia seeds as a source of natural lipid antioxidants. J. Amer. Oil Chem. Soc. 61, 928-931

Ubando-Rivera J., Navarro-Ocaña A., Valdivia-López M.A., 2005. Mexican lime peel: Comparative study on contents of dietary fibre and associated antioxidant activity. Food Chem. 89, 57-61

Vasta V., Nudda A., Cannas A., Lanza M., Priolo A., 2008. Alternative feed resources and their effects on the quality of meat and milk from small ruminants. Anim. Feed Sci. Tech. 147, 223-246

Velázquez-Avendaño J., Perezgrovas-Garza R., Velasco-Zebadúa M.E., Zaragoza-Martínez L., Rodríguez-Galván G., 2005. Assesment of quebracho (Acacia farnesiana) pods utilization in wool-sheep feeding. Arch. Zootec. 54, 535-540

Vemuri M., Kelley D., Erickson K.L., 2008. III chapter. Health effects of foods rich in polyphenols. In: F. De Meester, R. Ross Watson (Editors), Wild-Type Food in Health Promotion and Disease Prevention. The Columbus concept., Humana Press, New Jersey. USA, p. 567

Villalba J.J., Provenza F.D., Manteca X., 2010. Links between ruminants' food preference and their welfare. Animal 4, 1240-1247

von Gadow A., Joubert E., Hansmann C.F., 1997. Comparison of the antioxidant activity of aspalathin with that of other plant phenols of rooibos tea (Aspalathus linearis), alpha-tocopherol, BHT, and BHA. J. Agr. Food Chem. 45, 632-638

Wrage N., Strodthoff J., Cuchillo H.M., Isselstein J., Kayser M., 2011. Phytodiversity of temperate permanent grasslands: ecosystem services for agriculture and livestock management for diversity conservation, Biodiv. Conserv. 20, 3317-3339 